ORIGINAL ARTICLE

\title{
Expression of tumor necrosis factor receptor 2 in human non-small cell lung cancer and its role as a potential prognostic biomarker
}

\author{
Yan Wen Zhang*, Qian Qian Chen* (D), Jie Cao*, Lei Qian Xu, Xin Tang, Juan Wang, Jing Zhang \& \\ Li Xia Dong \\ Respiratory Department, Tianjin Medical University General Hospital, Tianjin, China
}

Keywords

Non-small cell lung cancer; prognosis; TNFR2.

\author{
Correspondence \\ Li Xia Dong, Respiratory Department, Tianjin \\ Medical University General Hospital, \#154 \\ Anshan Road, Tianjin 300052, China. \\ Tel: +8613001385918 \\ Fax: +862260361720 \\ Email: luckydonglixia@163.com
}

*These authors contributed equally to this work.

Received: 19 November 2018; Accepted: 27 November 2018.

doi: $10.1111 / 1759-7714.12948$

Thoracic Cancer 10 (2019) 437-444

\begin{abstract}
Background: Tumor necrosis factor receptor 2 (TNFR2) promotes tumor cell proliferation, activates immunosuppressive cells, and supports immune escape. However, its role in non-small cell lung cancer (NSCLC) has not been reported. Methods: Quantitative real-time PCR and Western blotting were used to evaluate TNFR2 in three NSCLC cell lines (A549, H1299, H1975) and normal lung epithelial cells (BEAS-2B). TNFR2 was evaluated in 71 tumor tissues and 25 adjacent normal lung tissues by immunohistochemistry and analyzed with respect to clinical parameters.

Results: The messenger RNA and protein levels of TNFR2 were significantly higher in A549, H1299, and H1975 cells than in BEAS-2B cells $(P<0.05)$ and differed significantly between NSCLC tissues and adjacent normal lung tissues by immunohistochemistry $(P<0.0001)$. TNFR2 is a independent prognostic factor in NSCLC. There have significantly differences in overall survival (OS) $(P=0.006)$ and disease-free survival (DFS) $(P=0.000)$ of NSCLC patients between TNFR2 low expression groups and TNFR2 high expression group.

Conclusion: TNFR2 is expressed in human NSCLC tissues and cell lines and is related to poor prognosis. TNFR2 may represent a new auxiliary index for patients with NSCLC.
\end{abstract}

\section{Introduction}

Lung cancer is the most common malignant tumor with the highest morbidity and mortality worldwide. It is estimated that approximately 154050 people will die of the disease worldwide in 2018. ${ }^{1}$ Notably, human non-small cell lung cancer (NSCLC) accounts for about $85 \%$ of all primary lung cancers. ${ }^{2,3}$ With the recent development of tumor immunotherapy, molecular pathways underlying NSCLC progression have been explored and targeted drugs have brought new hope to some patients with NSCLC. However, the five-year survival rate is still very low at approximately $18 \%{ }^{1}$ Therefore, it is necessary to identify new and more effective therapeutic targets for NSCLC.

Both tumor necrosis factor receptor 1 (TNFR1) and TNFR2 are receptors of TNF. These two receptors trigger divergent signaling pathways upon interaction with TNF because of their different cytoplasmic domains. TNFR1 signaling pathways have been extensively characterized. They mediate the cytotoxic effects of TNF and induce cell apoptosis signaling pathways by activating the death domain in its cytoplasmic region, ${ }^{4}$ which may lead to potent antitumor effects. However, compared to TNFR1, few TNFR2 signaling pathways have been elucidated. TNFR2 is predominantly expressed in $\mathrm{T}$ lymphocytes, myeloid-derived suppressor cells, endothelial cells, myocardial cells, oligodendrocytes, and thymic cells, with important roles in antiinflammatory processes and immune regulation. Reports of TNFR2 functions in tumor progression have recently increased. TNFR2 has been found in various cancers, such as ovarian, ${ }^{5,6}$ breast, $^{7}$ esophageal, ${ }^{8}$ myeloma, ${ }^{9}$ and colorectal cancer. ${ }^{10}$ TNFR2 is considered a tumor-promoting factor and participates in tumor progression by promoting tumor cell proliferation, activating immunosuppressive cells, and 
supporting immune escape. Hence, TNFR2 may represent a potential target for cancer therapy.

Although a previous study showed that the loss of TNFR2 results in TNF-associated tumor cell death and tumor growth in a lung cancer mouse model, studies of TNFR2 in human NSCLC are lacking. ${ }^{11}$ In this study, we evaluated the expression of TNFR2 in human NSCLC tissues and cell lines and analyzed its relationship with clinicopathological parameters and survival outcomes. These results provide a basis for studies of the mechanism underlying the effects of TNFR2 in tumor progression. Additionally, this study provides preliminary evidence of the role of TNFR2 in human NSCLC and may contribute to the development of new cancer therapies.

\section{Methods}

\section{Cell culture}

BEAS-2B cells were obtained from the Laboratory of Lung Development and Diseases at Nankai University (Tianjin, China). A549, H1299, and H1975 cell lines were obtained from the Institute of Lung Cancer, Tianjin Medical University General Hospital (Tianjin, China). BEAS-2B cells were cultured in Dulbecco's modified Eagle medium and A549, H1299, and H1975 cells were cultured in RPMI 1640 supplemented with 10\% fetal bovine serum (Gibco, Carlsbad, CA, USA) and antibiotics in a $37^{\circ} \mathrm{C}$ incubator with a humidified atmosphere containing $5 \% \mathrm{CO}_{2}$.

\section{Quantitative real-time PCR}

RNA was isolated using TRIzol according to the manufacturer's instructions and reverse-transcribed into complementary DNA using Moloney Murine Leukemia Virus reverse transcriptase. Complementary DNA was amplified by real-time fluorescence quantitative PCR to detect expression levels. The expression level of TNFR2 was determined using a SYBR Green Master Mix kit (Roche Diagnostics, Indianapolis, IN, USA). The $\beta$-actin gene was used as an internal control to correct the expression of the target gene. The same reaction was performed three times, and quantitative real-time (qRT) PCR results were evaluated using the $2^{-\nabla \nabla} \mathrm{CT}$ method. The primers used in this study were as follows: TNFR2, forward $5^{\prime}$ TTCATCCACGGATATTTGCAGG- $3^{\prime}$ and reverse $5^{\prime}$ AGGCCAACCGTGGAAAAGATG- $3^{\prime}$; $\beta$-actin, forward $5^{\prime}$-AGGCCAACCGTGAAAAGATG- $3^{\prime}$ and reverse $5^{\prime}$ AGAGCATAGCCCTCGTAGATGG-3'.

\section{Western blot analysis}

Proteins were extracted from tumor cell lines. The protein concentration was measured using a BCA Assay Kit (Pierce Biotechnology, Rockford, IL, USA). Proteins were separated by sodium dodecyl sulfate-polyacrylamide gel electrophoresis and electroblotted onto Nitrocellulose membranes (Millipore, Bedford, MA, USA). The membranes were blocked with 5\% skim milk for two hours at room temperature and then incubated overnight at $4{ }^{\circ} \mathrm{C}$ with the primary antibody. Primary anti-TNFR2 (1:1000; Proteintech Group Inc., Chicago, IL, USA) and $\beta$-tubulin (1:1000; Santa Cruz Biotechnology Inc., Santa Cruz, CA, USA) were used to probe the target proteins. Goat antirabbit immunoglobulin G-horseradish peroxidase (1:5000; Santa Cruz Biotechnology Inc.) was used as a secondary antibody and incubated with the membrane for two hours at room temperature. The bands were observed with an electrochemiluminescence reagent. The intensity of the protein bands on the scanned images was analyzed using the Tanon GIS digital image analysis system (Tanon, Shanghai, China). The cumulative TNFR2 level was quantified as the ratio of the band intensity of the target protein to $\beta$-tubulin.

\section{Tissues and patients}

Paraffin-embedded tissues (71 NSCLC and 25 adjacent normal tissues) were acquired for immunohistochemistry (IHC) from patients who underwent surgery from January 2006 to December 2010 at Tianjin Medical University General Hospital. The ethics committee and review board approved the study. Patients were not administered radiotherapy, chemotherapy, or immunotherapy prior to surgery. Patient information is shown in Table 1.

\section{Immunohistochemistry (IHC)}

After paraffin tissue sectioning, samples were placed in an oven at $55^{\circ} \mathrm{C}$ for 60 minutes. The dried tissue sections were dewaxed into water. The dewaxing process was as follows: xylene, 10 minutes; xylene, 10 minutes; absolute ethanol, 10 minutes; anhydrous ethanol 10 minutes: $95 \%$ ethanol 10 minutes; 95\% ethanol 10 minutes; $85 \%$ ethanol 10 minutes; $70 \%$ ethanol 10 minutes; and tap water. The antigens were then recovered by high-pressure heating with citrate buffer (Maixin Biotechnology Development Co., Ltd., Fuzhou, China) and $3 \% \mathrm{H}_{2} \mathrm{O}_{2}$ for 10 minutes, blocked with $5 \%$ normal goat serum (NGS) at room temperature for one hour, and incubated with a NGS-diluted TNFR2 antibody (dilution, 1:300; Proteintech Group Inc.) overnight at $4^{\circ} \mathrm{C}$. NGS was used as a negative control. Samples were incubated with biotin-labeled goat anti-mouse/rabbit 
Table 1 Association between TNFR2 expression level and clinicopathological characteristics of NSCLC

\begin{tabular}{|c|c|c|c|c|}
\hline \multirow[b]{2}{*}{ Clinical parameters } & \multirow[b]{2}{*}{ Cases } & \multicolumn{2}{|l|}{ TNFR2 } & \multirow[b]{2}{*}{$P$} \\
\hline & & (high) & (low) & \\
\hline Age (year) & 71 & 25 & 46 & \\
\hline$\leq 65$ & 43 & 16 & 27 & \\
\hline$>65$ & 28 & 9 & 19 & 0.662 \\
\hline \multicolumn{5}{|l|}{ Gender } \\
\hline Male & 41 & 16 & 25 & \\
\hline Female & 30 & 9 & 21 & 0.432 \\
\hline \multicolumn{5}{|l|}{ Tumor size } \\
\hline$\leq 5 \mathrm{~cm}$ & 63 & 23 & 40 & \\
\hline$>5 \mathrm{~cm}$ & 8 & 2 & 6 & 0.521 \\
\hline \multicolumn{5}{|c|}{ Histological type degree } \\
\hline Squamous & 25 & 7 & 18 & \\
\hline Adenocarcinoma & 41 & 17 & 24 & \\
\hline Other & 5 & 1 & 4 & 0.411 \\
\hline \multicolumn{5}{|l|}{ Clinical stage } \\
\hline$|/| \mid$ & 51 & 13 & 38 & \\
\hline III/IV & 20 & 12 & 8 & $0.006^{*}$ \\
\hline \multicolumn{5}{|c|}{ Regional lymph node involvement } \\
\hline No & 45 & 11 & 34 & \\
\hline Yes & 26 & 14 & 12 & $0.012^{*}$ \\
\hline
\end{tabular}

$\star P<0.05$ indicates statistical significance. NSCLC, non-small cell lung cancer; TNFR2, tumor necrosis factor receptor 2 .

immunoglobulin G (Maixin Biotechnology Development Co., Ltd.) for 15 minutes the next day and then incubated and developed using diaminobenzidine solution (Maixin Biotechnology Development Co., Ltd.). Hematoxylin was used for counterstaining for two minutes and neutral gum was used for sealing. Two pathologists blindly analyzed any discrepancies to avoid bias. Positive cells were scored based on percentages as follows: $0=0 \%, 1=\leq 25 \%, 2=26-75 \%$, and $3=>75 \%$. Staining was ranked by intensity as follows: $0=$ no staining, $1=$ weak staining, 2 moderate staining, and $3=$ intense staining. Finally, TNFR2 expression was evaluated by the product of the percentage of positive cells and the intensity of staining. TNFR2 expression was divided into two categories: low $(<4)$ and high $(\geq 4)$.

\section{Statistical analyses}

SPSS version 23.0 (IBM Corp., Armonk, NY, USA) was used for statistical analyses. Chi-square tests were used to analyze the correlation between TNFR2 and clinical parameters. Kaplan-Meier curves were drawn, and logrank tests were used to verify the relationship between TNFR2 expression and prognosis. A Cox proportional hazards regression model was used to identify factors affecting the OS and DFS rates in NSCLC. $P<0.05$ indicated a statistically significant difference.

\section{Results}

\section{Tumor necrosis factor receptor 2 (TNFR2) expression in human non-small cell lung cancer (NSCLC) cell lines and tissue samples}

TNFR2 messenger RNA (mRNA) and protein expression levels were assessed by qRT-PCR and Western blotting, respectively. According to qRT-PCR analysis, the expression levels of TNFR2 mRNA in human NSCLC cell lines, including A549, H1299, and H1975, were significantly higher than those in the normal human lung epithelial cell line, BEAS-2B (Fig 1a) $(P<0.05)$. The average relative levels of TNFR2 mRNA expression in the human NSCLC cell lines A549,
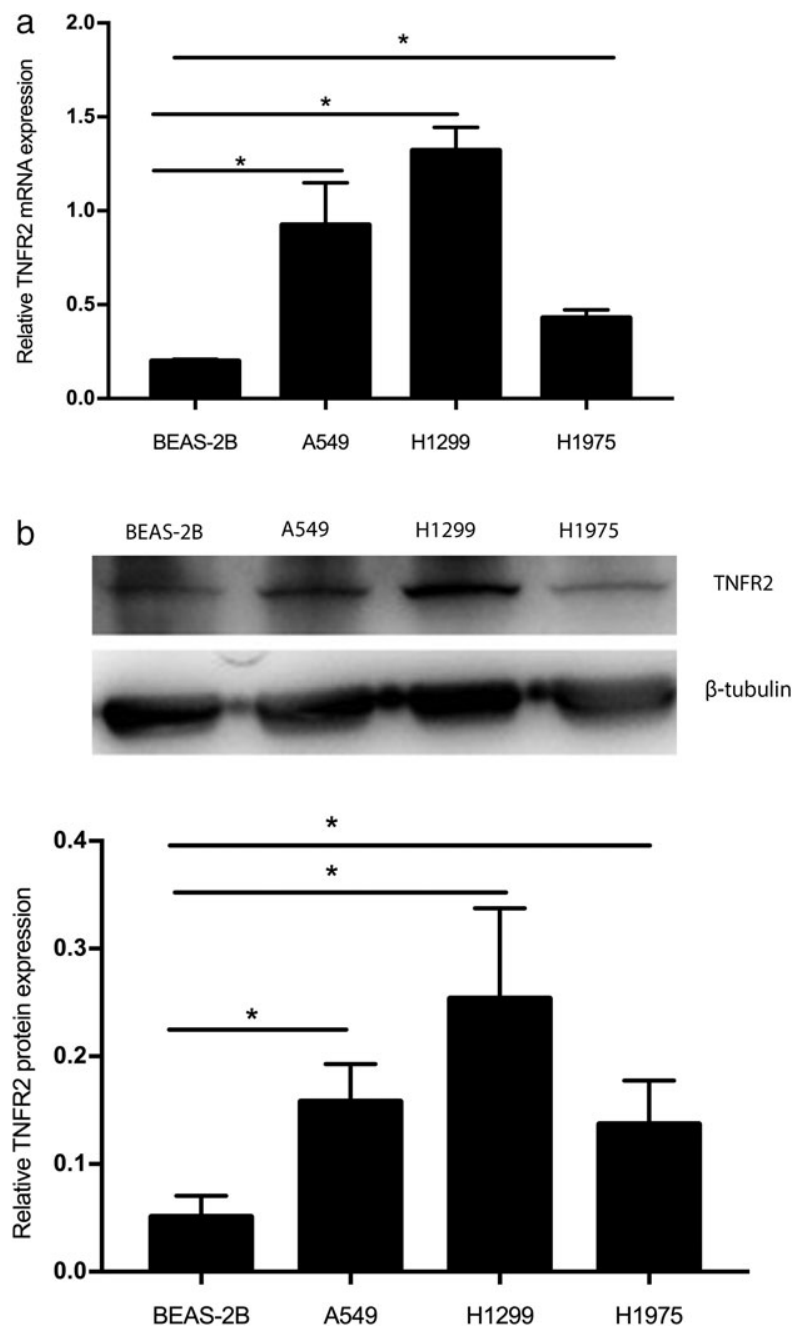

Figure 1 Expression of tumor necrosis factor receptor 2 (TNFR2) in human non-small cell lung cancer (NSCLC) cell lines (A549, H1299, H1975) and normal lung epithelial cells (BEAS-2B) was analyzed by (a) real time-PCR at messenger RNA (mRNA) level and (b) Western Blot analysis at protein level and mean of triplicate assays. ${ }^{*} P<0.05$. 
H1299, and H1975 were $0.9237 \pm 0.1302,1.322 \pm 0.07141$, and $0.4297 \pm 0.02441$, respectively, which were significantly higher than that in the BEAS-2B cell line $(0.2 \pm 0.005774)$ $(P<0.05)$. Furthermore, Western blot analysis indicated that compared to the normal human lung epithelial cell line, the TNFR2 protein expression levels in human NSCLC cell lines were much higher (Fig 1b) $(P<0.05)$.

Additionally, 71 primary human NSCLC specimens were collected to evaluate the expression of TNFR2 in cancer tissues by IHC. Both adenocarcinoma (ADC) and squamous cell carcinoma (SCC) cells from paraffinembedded NSCLC tissues exhibited deep staining. TNFR2 expression was detected predominantly in cell membrane and cytoplasm (Fig 2a), but weak staining was also observed in cancer stromal cells. TNFR2 expression was detected in both lung ADC (Fig 2c,d) and lung SCC (Fig 2e,f) tissue samples by IHC. The rate of high TNFR2 expression in all 71 primary human NSCLC specimens was $35 \%$. However, TNFR2 expression in 25 adjacent normal lung tissue samples was very low: 6 cases showed low expression while the remaining 19 showed negative expression (Fig 2b).

\section{Correlation between TNFR2 expression and clinical parameters in patients with NSCLC}

As shown in Table 1, high TNFR2 expression was associated with tumor stage $(P=0.006)$ and lymph node metastasis $(P=0.012)$. Based on TNFR2 expression, 71 NSCLC specimens were divided into two groups: low $(n=46)$ and high $(n=25)$ expression. Eight out of 46 patients in the low TNFR2 group were classified as stage III/IV, which was significantly lower than the proportion in the high expression group (12 of 25 patients, $P=0.006$ ). Twelve out of 46 patients in the low TNFR2 group had lymph node metastasis, which was significantly lower than the proportion in the high TNFR2 group (14 of 25 patients, $P=0.012$ ). However, differences in age, gender, and tumor size between the two groups were not significant. These results confirm associations between TNFR2 and clinical stage and lymph node metastasis.

\section{TNFR2 protein expression predicts NSCLC survival}

TNFR2 was positively associated with poor prognosis. In patients with NSCLC with high TNFR2 expression, the a

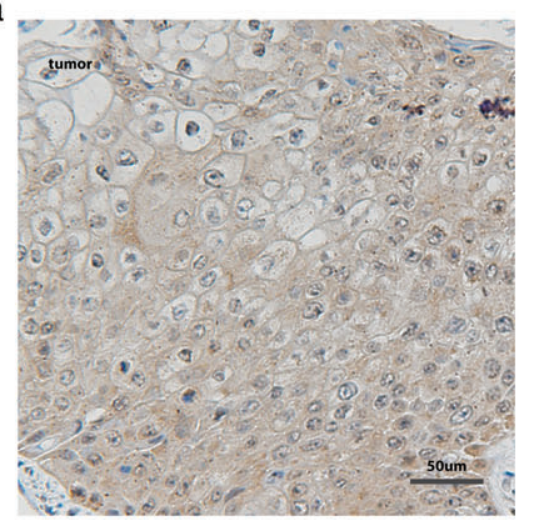

b

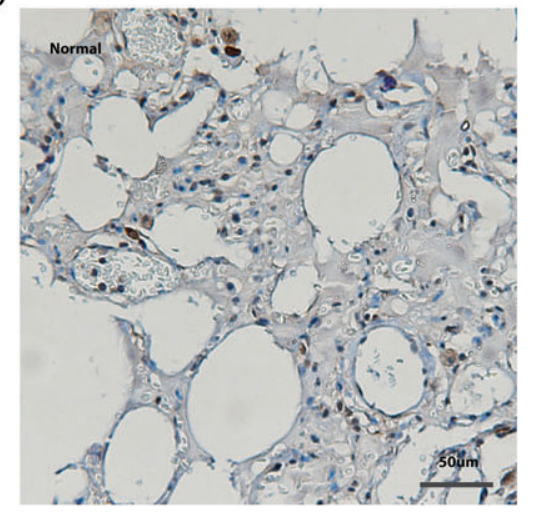

C

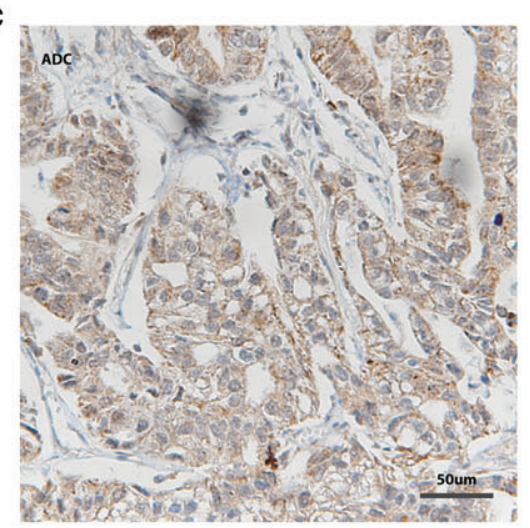

d

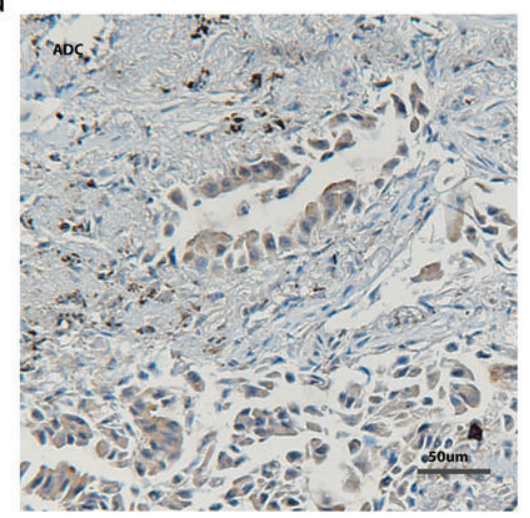

e

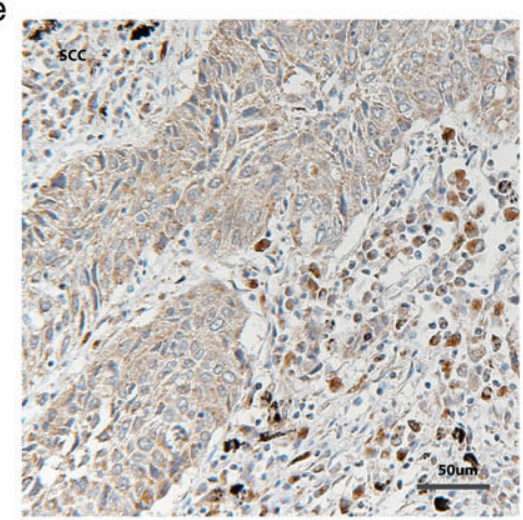

f

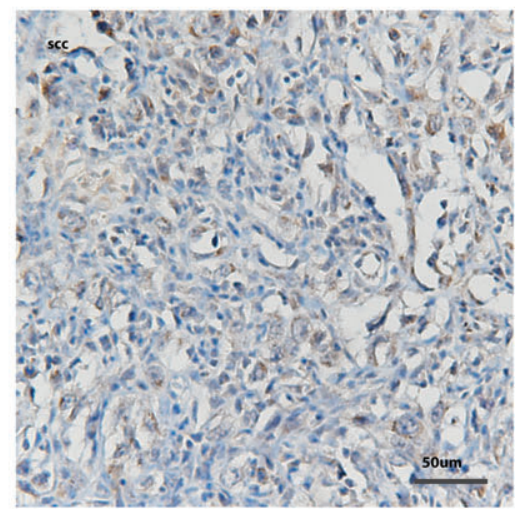

Figure 2 Expression of tumor necrosis factor receptor 2 (TNFR2) in (a) human non-small cell lung cancer (NSCLC) specimens and (b) adjacent normal lung tissues. (c) High and (d) low expression (x200) in lung adenocarcinoma and (e) high and (f) low expression (x200) in lung squamous cell carcinoma, respectively. 

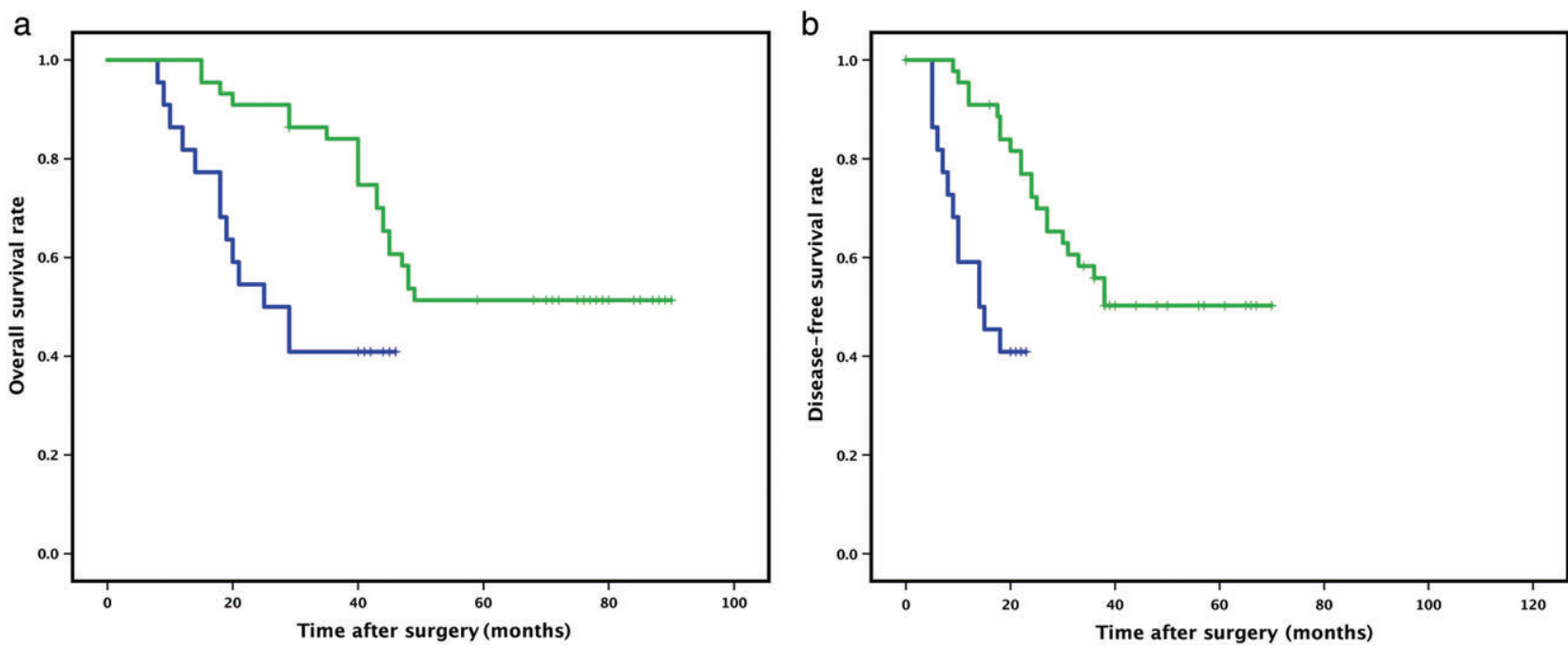

Figure 3 High tumor necrosis factor receptor 2 (TNFR2) expression was associated with shorter survival time in non-small cell lung cancer (NSCLC) patients. (a) Overall and (b) disease-free survival in tumor tissues of NSCLC patients with high and low TNFR2 expression was calculated using Kaplan-Meier survival analysis. ${ }^{*} P<0.05$.

mean OS and DFS were 29 and 15 months compared to 64 and 47 months for patients with low TNFR2 expression, respectively. Survival rates were estimated using the Kaplan-Meier method and differences were compared using a log-rank test. Differences in OS $(P=0.006)$ and DFS $(P=0.000)($ Fig 3$)$ between the two groups were significant (Fig 2).

We assessed whether TNFR2 expression is an independent prognostic factor for OS or DFS by univariate and multivariate Cox regression analyses (Tables 2-3). Univariate analysis showed that the key indicators of poor OS were clinical stage, lymph node metastasis, and TNFR2 expression. Multivariate analysis confirmed that TNFR2 (hazard ratio [HR] 2.332, 95\% confidence interval [CI] 1.057-5.144; $P=0.036)$, clinical stage (HR $0.039,95 \%$ CI $0.014-0.106$; $P=0.000$ ), lymph node metastasis (HR 1.142, 95\% CI $0.382-3.413 ; \quad P=0.000)$, and adenocarcinoma subtype (HR $0.244, \quad 95 \%$ CI $0.064-0.926 ; \quad P=0.039$ ) were independent predictors of OS. In addition, the results of the univariate analysis confirmed that the key predictors of poor DFS included clinical stage, lymph node metastasis, and TNFR2 expression. The results of multivariate analysis showed that TNFR2 (HR 4.487, 95\% CI 1.761-11.431; $P=0.002)$ and clinical stage (HR $0.028,95 \%$ CI $0.009-0.089 ; \quad P=0.000)$ were independent predictors of DFS.

The univariate and multivariate Cox regression analyses further indicated the significant function of TNFR2 as an independent prognostic factor in NSCLC. The results further revealed that high TNFR2 expression was associated with poor prognosis in patients with NSCLC.

\section{Discussion}

Tumor necrosis factor- $\alpha$ (TNF- $\alpha$ ) binds to TNFR1 to promote cytotoxicity and TNFR2 to promote survival. A series

Table 2 Univariate and multivariate analyses identified factors affecting OS of patients with NSCLC

\begin{tabular}{|c|c|c|c|c|c|c|}
\hline \multirow[b]{2}{*}{ Clinical parameters } & \multicolumn{3}{|c|}{ Univariate analysis } & \multicolumn{3}{|c|}{ Multivariate analysis } \\
\hline & $H R$ & $95 \% \mathrm{Cl}$ & $P$ & $\mathrm{HR}$ & $95 \% \mathrm{Cl}$ & $P$ \\
\hline TNFR2 & 2.371 & $1.135-4.953$ & $0.022 *$ & 2.332 & $1.057-5.144$ & $0.036^{*}$ \\
\hline Gender & 1.050 & $0.530-2.079$ & 0.890 & 0.521 & $0.236-1.153$ & 0.108 \\
\hline Age (year) & 0.908 & $0.455-1.816$ & 0.787 & 1.203 & $0.541-2.677$ & 0.650 \\
\hline Tumor size & 2.195 & $0.907-5.312$ & 0.081 & 1.425 & $0.466-4.360$ & 0.535 \\
\hline Clinical stage & 0.078 & $0.036-0.167$ & $0.000 *$ & 0.039 & $0.014-0.106$ & $0.000^{*}$ \\
\hline Regional lymph node involvement & 0.229 & $0.113-0.465$ & $0.000 *$ & 1.142 & $0.382-3.413$ & $0.000 *$ \\
\hline \multicolumn{7}{|l|}{ Histological type degree } \\
\hline Adenocarcinoma & 0.643 & $0.189-2.187$ & 0.480 & 0.244 & $0.064-0.929$ & $0.039 *$ \\
\hline Squamous & 0.684 & $0.195-2.405$ & 0.554 & 0.906 & $0.243-3.375$ & 0.883 \\
\hline
\end{tabular}

$\star P<0.05$ was considered statistically significant. $\mathrm{Cl}$, confidence interval; HR, hazard ratio; NSCLC, non-small cell lung cancer; OS, overall survival. 
Table 3 Univariate and multivariate analyses identified factors affecting DFS of patients with NSCLC

\begin{tabular}{|c|c|c|c|c|c|c|}
\hline \multirow[b]{2}{*}{ Clinical parameters } & \multicolumn{3}{|c|}{ Univariate analysis } & \multicolumn{3}{|c|}{ Multivariate analysis } \\
\hline & $H R$ & $95 \% \mathrm{Cl}$ & $P$ & $H R$ & $95 \% \mathrm{Cl}$ & $P$ \\
\hline TNFR2 & 3.724 & $1.612-8.602$ & $0.002^{*}$ & 4.487 & $1.761-11.431$ & $0.002 *$ \\
\hline Gender & 1.021 & $0.516-2.203$ & 0.952 & 0.483 & $0.220-1.059$ & 0.069 \\
\hline Age (year) & 0.885 & $0.443-1.768$ & 0.729 & 1.041 & $0.470-2.301$ & 0.922 \\
\hline Tumor size & 1.911 & $0.789-4.631$ & 0.151 & 1.413 & $0.469-4.260$ & 0.539 \\
\hline Clinical stage & 0.073 & $0.034-0.160$ & $0.000 *$ & 0.028 & $0.009-0.089$ & $0.000 *$ \\
\hline $\begin{array}{l}\text { Regional lymph node involvement } \\
\text { Histological type degree }\end{array}$ & 0.239 & $0.119-0.479$ & $0.000^{*}$ & 0.810 & $0.296-2.217$ & 0.681 \\
\hline Adenocarcinoma & 0.781 & $0.229-2.666$ & 0.694 & 0.277 & $0.072-1.067$ & 0.062 \\
\hline Squamous & 0.783 & $0.223-2.751$ & 0.702 & 1.444 & $0.375-5.561$ & 0.594 \\
\hline
\end{tabular}

${ }^{\star} P<0.05$ was considered statistically significant. $\mathrm{Cl}$, confidence interval; DFS, disease-free survival; HR, hazard ratio; NSCLC, non-small cell lung cancer.

of studies have shown that TNFR2 plays important roles in the development and progression of various types of tumors, including breast, esophageal, cervical, ovarian, and colorectal cancers, myeloma, cholangiocarcinoma, and non-Hodgkin's lymphoma., ${ }^{9,12-14}$ A previous study showed that tumor growth is significantly reduced in TNFR2 knockout Lewis lung cancer mice; ${ }^{11}$ it is possible that TNF signaling increases tumor and endothelial cell death via TNFR1 and not via TNFR2. However, to our knowledge, the role of TNFR2 has not been evaluated in human NSCLC tissues and cell lines.

In this study, we analyzed TNFR2 expression in human NSCLC cell lines for the first time. We detected TNFR2 expression in three human NSCLC cell lines. TNFR2 expression levels in the human NSCLC cell lines A549, H1299, and H1975 were approximately 4.6, 6.61, and 2.15 times higher at the mRNA level and 3.1, 4.99, and 2.69 times higher at the protein level than in the BEAS-2B cell line, respectively. Compared to the BEAS-2B cell line, TNFR2 expression levels in human NSCLC cell lines were significantly higher at both the mRNA and protein expression levels. These results suggest that TNFR2 plays an important role in human NSCLC progression.

To further verify the expression of TNFR2 in human NSCLC, we examined its expression in human NSCLC tissues. Immunohistochemical staining showed that TNFR2 is mainly located in the cytoplasm and membranes, and weak staining was observed in cancer stromal cells. These results provide the first evidence of the expression of TNFR2 in human NSCLC tissues. Additionally, the tumor stromal localization of TNFR2 is consistent with previous results concerning TNFR2 expression in regulatory $\mathrm{T}$ cells (Tregs). We then analyzed the relationship between TNFR2 expression and clinical parameters and prognosis in patients with NSCLC. We found correlations between TNFR2 expression and both clinical stage and lymph node metastasis.
To examine the prognostic significance of TNFR2 expression in human NSCLC, we constructed OS curves. Unsurprisingly, we found that higher TNFR2 expression in human NSCLC tissues indicates a shorter survival time. In patients with NSCLC with high TNFR2 expression, the mean OS and DFS were 29 and 15 months compared to 64 and 47 months for patients with low TNFR2 expression, respectively. Hence, TNFR2 may be a new auxiliary indicator for predicting the clinical prognosis of patients with NSCLC.

Recent advances in high-throughput sequencing technology and a greater understanding of tumor cell biology have led to the development of targeted therapies for human NSCLC. ${ }^{15-17}$ We found that TNFR2 is expressed in human NSCLC tissues and cells, and its expression is significantly correlated to poor prognosis. Thus, we speculated that TNFR2 may be involved in several signaling pathways to promote malignant biological behavior, such as proliferation, migration, and angiogenesis. However, further studies are needed to explore the molecular mechanisms by which TNFR2 promotes cancer progression.

A recent study showed that the level of TNFR2 expressed by Tregs is significantly higher than that of conventional $\mathrm{T}$ cells in the peripheral blood of patients with lung cancer, and TNFR2 levels on Tregs are associated with the number and activity of Tregs, as well as lymphatic invasion, distant metastasis, and a more advanced clinical stage. ${ }^{18}$ Tregs have a central role in regulating tumorspecific immune responses. ${ }^{19-21}$ TNFR2 stimulates the activation and proliferation of Tregs, thereby reducing the ability to resist tumor immune responses, affecting the promotion of tumor cell survival and tumor growth. ${ }^{22-26}$ Chen et al. and Torrey et al. both found that blocking TNFR2 may enhance the antitumor immune response and directly kill tumor cells. ${ }^{25,27}$ In this study, we detected TNFR2 expression in human NSCLC cells and tissues. The expression level of TNFR2 in human NSCLC was associated with 
advanced clinical stage and lymph node metastasis, as well as poor prognosis. The expression of TNFR2 in cancer cells and Tregs indicates that TNFR2 may be a new target for human NSCLC and a novel checkpoint for immunotherapy. TNFR2 inhibitors may be able to improve the efficacy of chemotherapy and radiation therapy in patients with NSCLC and prolong survival.

However, because the tissue samples in this experiment were obtained from patients in Tianjin, the experimental data were clearly regional. The number of samples was relatively small; accordingly, the results may not be generalizable to all patients with NSCLC. Multi-center experiments are needed to expand the sample size and validate our results.

In conclusion, we detected the expression of TNFR2 in human NSCLC cells and tissues and found that higher TNFR2 expression in human NSCLC leads to a more advanced clinical stage and shorter survival. These findings suggest that TNFR2 inhibitors may be effective for patients with NSCLC and have the potential to become a new adjuvant therapy. Our results suggest that TNFR2 is a useful prognostic marker for patients with NSCLC. Because the antitumor function of TNFR2 is not fully resolved, a deeper understanding of its mechanism of action may be useful to establish effective tumor immunotherapies.

\section{Acknowledgments}

This study was supported by the National Natural Science Foundation of China (Grant no. 81600067), the National Natural Science Foundation of China (Grant no. 81670084) and the youth incubation fund of General Hospital Affiliated to Tianjin Medical University (ZYYZY2016016).

\section{Disclosure}

No authors report any conflict of interest.

\section{References}

1 Siegel RL, Miller KD, Jemal A. Cancer statistics, 2018. CA Cancer J Clin 2018; 68: 7-30.

2 Midha A, Dearden S, McCormack R. EGFR mutation incidence in non-small cell lung cancer of adenocarcinoma histology: A systematic review and global map by ethnicity (mutMapII). Am J Cancer Res 2015; 5: 2892-911.

3 Zago G, Muller M, van den Heuvel M, Baas P. New targeted treatments for non-small-cell lung cancer-Role of nivolumab. Biologics 2016; 10: 103-17.

4 Miao X, Huang Y, Liu TT et al. TNF- $\alpha /$ TNFR1 signaling is required for the full expression of acute and chronic itch in mice via peripheral and central mechanisms. Neurosci Bull 2018; 34: 42-53.
5 Ferrarelli LK. Locking TNFR2 to kill ovarian cancer. Science 2017; 355: 257-8.

6 Chen X, Oppenheim JJ. Targeting TNFR2, an immune checkpoint stimulator and oncoprotein, is a promising treatment for cancer. Sci Signal 2017; 10: eaal2328.

7 Yang F, Zhao N, Wu N. TNFR2 promotes Adriamycin resistance in breast cancer cells by repairing DNA damage. Mol Med Rep 2017; 16: 2962-8.

8 Yang D, Li R, Wang $\mathrm{H}$ et al. Clinical significance of tumor necrosis factor receptor 2 in middle and lower thoracic esophageal squamous cell carcinoma. Oncol Lett 2018; 16: 2971-8.

9 Grotowski M, CEA WS. CA199 and il-8, sTNFRII and sil$2 \mathrm{R}$ in persons at high risk of colorectal cancer. Pol Merkur Lekarski 2003; 14: 327-30.

10 Heemann C, Kreuz M, Stoller I et al. Circulating levels of TNF receptor II are prognostic for patients with peripheral T-cell non-Hodgkin lymphoma. Clin Cancer Res 2012; 18: 3637-47.

11 PLOS ONE Staff. Correction: Therapeutic non-toxic doses of TNF induce significant regression in TNFR2-p75 knockdown Lewis lung carcinoma tumor implants. PLoS One 2014; 9: e92373.

12 Arnott CH, Scott KA, Moore RJ, Robinson SC, Thompson RG, Balkwill FR. Expression of both TNF-alpha receptor subtypes is essential for optimal skin tumour development. Oncogene 2004; 23: 1902-10.

13 Tanimura Y, Kokuryo T, Tsunoda $\mathrm{N}$ et al. Tumor necrosis factor alpha promotes invasiveness of cholangiocarcinoma cells via its receptor, TNFR2. Cancer Lett 2005; 219: 205-13.

14 Jöhrer K, Janke K, Krugmann J, Fiegl M, Greil R. Transendothelial migration of myeloma cells is increased by tumor necrosis factor (TNF)-alpha via TNF receptor 2 and autocrine up-regulation of MCP-1. Clin Cancer Res 2004; 10: 1901-10.

15 Marrone KA, Naidoo J, Brahmer JR. Immunotherapy for lung cancer: No longer an abstract concept. Semin Respir Crit Care Med 2016; 37: 771-82.

16 Hirsch FR, Suda K, Wiens J, Bunn PA Jr. New and emerging targeted treatments in advanced non-small-cell lung cancer. Lancet 2016; 388: 1012-24.

17 National Comprehensive Cancer Network. NCCN Clinical Practice Guidelines in Oncology: Non-small Cell Lung Cancer. Version 5. 2017, 2017.

18 Yan F, Du R, Wei F et al. Expression of TNFR2 by regulatory $\mathrm{T}$ cells in peripheral blood is correlated with clinical pathology of lung cancer patients. Cancer Immunol Immunother 2015; 64: 1475-85.

19 Facciabene A, Motz GT, Coukos G. T-regulatory cells: Key players in tumor immune escape and angiogenesis. Cancer Res 2012; 72: 2162-71.

20 Cari L, Nocentini G, Migliorati G, Riccardi C. Potential effect of tumor-specific Treg-targeted antibodies in the treatment of human cancers: A bioinformatics analysis. Oncoimmunology 2018; 7: e1387705. 
21 Byrne WL, Mills KH, Lederer JA, O’Sullivan GC. Targeting regulatory T cells in cancer. Cancer Res 2011; 71: 6915-20.

22 Vanamee ES, Faustman DL. TNFR2: A novel target for cancer immunotherapy. Trends Mol Med 2017; 23: 1037-46.

23 Chen X, Baumel M, Mannel DN, Howard OM, Oppenheim JJ. Interaction of TNF with TNF receptor type 2 promotes expansion and function of mouse CD4+CD25+ T regulatory cells. J Immunol 2007; 179: 154-61.

24 Nguyen DX, Ehrenstein MR. Anti-TNF drives regulatory T cell expansion by paradoxically promoting membrane TNFTNF-RII binding in rheumatoid arthritis. J Exp Med 2016;

213: $1241-53$.
25 Chen X, Subleski JJ, Kopf H, Howard OM, Mannel DN, Oppenheim JJ. Cutting edge: Expression of TNFR2 defines a maximally suppressive subset of mouse CD4+CD25+FoxP3+ $\mathrm{T}$ regulatory cells: Applicability to tumor-infiltrating $\mathrm{T}$ regulatory cells. J Immunol 2008; 180: 6467-71.

26 Chen X, Subleski JJ, Hamano R, Howard OM, Wiltrout RH, Oppenheim JJ. Co-expression of TNFR2 and CD25 identifies more of the functional CD4+FOXP3+ regulatory $\mathrm{T}$ cells in human peripheral blood. Eur J Immunol 2010; 40: 1099-106.

27 Torrey H, Butterworth J, Mera T et al. Targeting TNFR2 with antagonistic antibodies inhibits proliferation of ovarian cancer cells and tumor-associated Tregs. Sci Signal 2017; 10: eaaf8608. 